\title{
Effect of moisture on the behaviour of an extruded clay paste VIGNES Jérémie $^{1, a}$, SCHMIDT Fabrice ${ }^{1, b}$, DUSSERRE Gilles ${ }^{1, c}$ and DALMASSO Jean Frédéric ${ }^{2, d}$
}

\author{
${ }^{1}$ Université de Toulouse ; Mines Albi, INSA, UPS, ISAE, ICA (Institut Clément ADER) ; Campus \\ Jarlard, F-81013 Albi cedex 09, France \\ ${ }^{2}$ CRED TERREAL, Route de Revel, 11400 CASTELNAUDARY \\ ajeremie.vignes@mines-albi.fr, bfabrice.schmidt@mines-albi.fr, 'cgilles.dusserre@mines-albi.fr, \\ jean-frederic.dalmasso@terreal.com
}

Keywords: Clay, moisture, free compression test, rheological behaviors, inverse analyse, forming simulation.

\begin{abstract}
In the industrial process, the moisture of the clay sheet obtained by extrusion and pressed to form a tile varies in time. It depends on the nature and the mixing of the raw materials during the production. In order to model and understand the influence of the moisture on the pressing step, it is necessary to determine the parameters of the rheological and tribological laws. A study of the rheological behaviour, based on free compression tests on cylinder samples, allowed to use an elasto-visco-plastic behaviour for the extruded clay paste. The different constitutive parameters were estimated by an inverse analysis based on the experimental force/displacement curves. The identification was performed with the optimisation algorithm implemented in the commercial software Forge $\mathbb{R}$ 2009. The influence of the water content in the paste on the rheological parameters was identified and fitted using linear models. To understand the influence of the moisture, we simulated a compression test, using Forge ${ }^{\circledR}$ involving the shaping of a tile lug. This geometry is representative of the state of stress during the pressing of the tile, in an area currently subjected to defects. The numerical model shows that an increase of eighteen percent of the moisture, allows to decrease by half the pressing force.
\end{abstract}

\section{Introduction}

The material used to manufacture clay tiles contain around 22 percent of water (in percent of dry weight). This water provides to the raw material some ductile properties and thus allows it to be formed and to keep the geometry. The significant variability of the process used to incorporate the water leads to a modification of the rheological behaviour of the clay paste, and makes difficult the stabilisation of the industrial performance (quality, costs, delivery times). The understanding of the scientific links between the moisture, the rheological properties of the clay paste, and the final product (structure, aspect, mechanical properties) is fundamental to guarantee a high yield of the manufacturing process. In this paper, we will present a study, which aimed to determine the influence of the moisture on the behaviour of a clay paste. The influence of the water on clay paste was often studied to determine the plasticity threshold [1,2] and in particular, the Atterberg's limits $[3,4]$ but rarely the influence of the moisture on the rheological behaviour. To identify the rheological behaviour, compression tests were often performed to identify the rheological parameters [5]. The main difficulty of this test is the determination of the friction behaviour between the clay and the compression tools [6]. To escape the unknown friction, we cover the tools with sandpaper [7]. The friction law can be identified from the compression tests with the measure of the evolution of the free surface of the sample. Djelal [8] has designed a rectilign tribometer to identify the friction between clay mixture an metallic tools. The purpose of this study is to understand the effect of the moisture on the pressing step of a clay tile. The first part of this paper will be devoted to the presentation of the material and the experimental setup. After, we will present the behaviour laws used and the parameters identified. In the last section, we will address numerical simulations of a tile lug, representative of defects encountered on an industrial tile. 


\section{Material presentation}

The studied material is an extruded clay paste. It is composed of clay, sand and water. The clay is the main component of the paste (around $75 \%$ of the dry weight). The sand is a component whose function is to limit the cracking during the drying and the firing step of the manufacturing process. During the firing step, the sand forms a network, which allows to give the final mechanical properties. The sand represents $25 \%$ of the dry weight. The water contained in the paste allows to obtain the plasticity of the clay (capacity to be formed without defects appearance) [2]. The Atterberg limits [9] give the minimum and the maximum percentage of water in order to obtain a plastic paste. For this study, the moisture varies between 19 and 25 percent of the dry weight. The moisture of the mixture is not the controlled parameter during the elaboration of the paste. Indeed, during the extrusion step, the pressure in the extrusion die is measured and the water content becomes an adjustment variable. This process parameter, easily measured using a pressure sensor, allows to estimate quickly the fluidity of the paste. Consequently, a low pressure is signifiant of a fluid paste (high moisture) and a high pressure represents a thick paste (low moisture). The extrusion pressure of the studied paste varies between five to ten bars.

\section{Experimental setup}

To identify the rheological behaviour of the clay paste, we performed compression tests using an electro-mechanical testing machine, equipped with a $30 \mathrm{kN}$ load cell. Cylindrical samples with a diameter of $60 \mathrm{~mm}$ and a height of $25 \mathrm{~mm}$ were used. The resulting distribution of stresses is very similar to those involved in the pressing step. In order to estimate the constitutive parameters using inverse analysis, we performed two types of compression profile. The first test, used for the identification of the behaviour, consists to compress the sample while imposing an increasing crosshead displacement rate. The second compression profile consists to a constant cross head displacement rate used with three different velocities $(0.1 \mathrm{~mm} / \mathrm{s}, 1 \mathrm{~mm} / \mathrm{s}$ and $8.5 \mathrm{~mm} / \mathrm{s})$. These tests allow to validate the rheological parameters identified with the first compression profile. The compression paths are plotted in the figure 1.

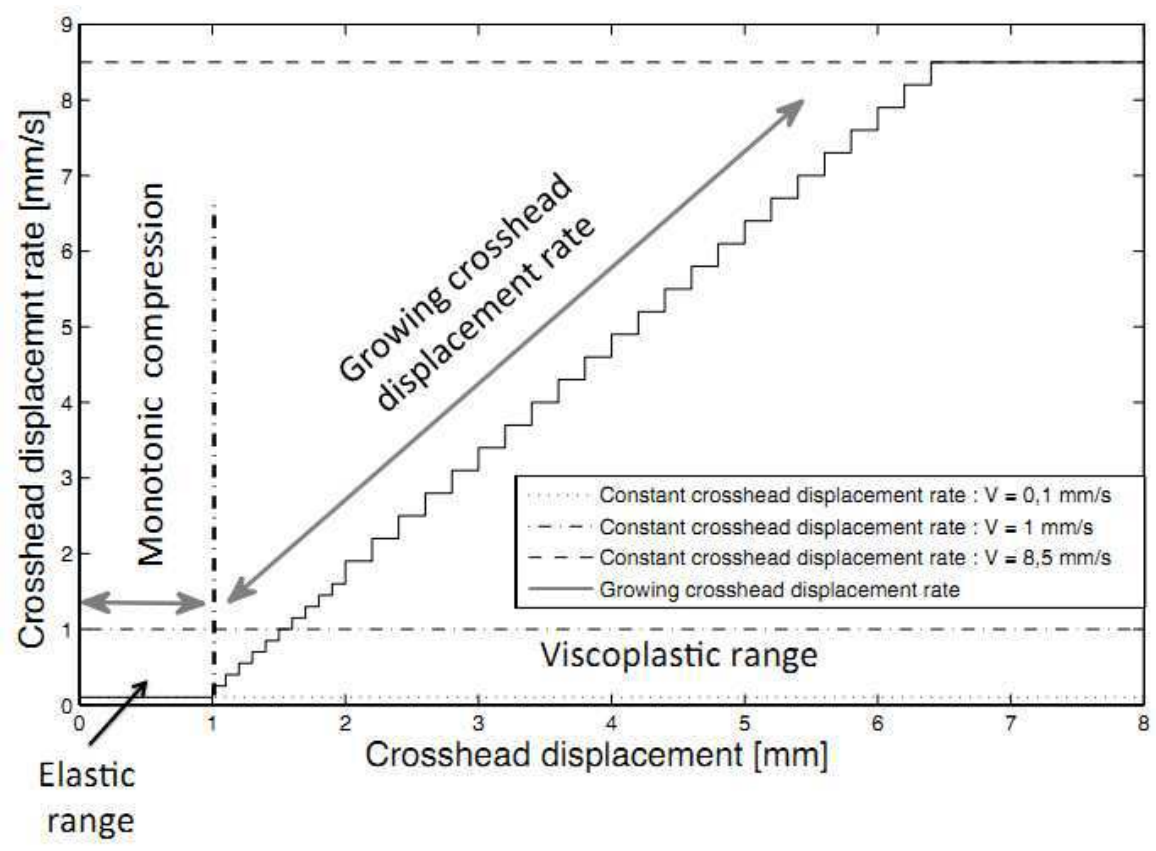

Figure 1: Different velocity paths used for the compression tests

The first profile was chosen to determine precisely the rheological parameters and in particular the sensitivity to strain rate only on one compression test in order to avoid the variabilities between two samples. The low speed on the first millimeter of compression allows to highlight the elastic part of 
the behaviour. The second part of the test, when the compression velocity increases, allows to identify rigorously the effect of the strain rate on the behaviour. The determination of the different parameters with a free compression test is difficult when the friction between the tools and the sample is unknown. This is why, we decided to cover the tools with sandpaper to create a quasisticky contact between the tools and the clay paste.

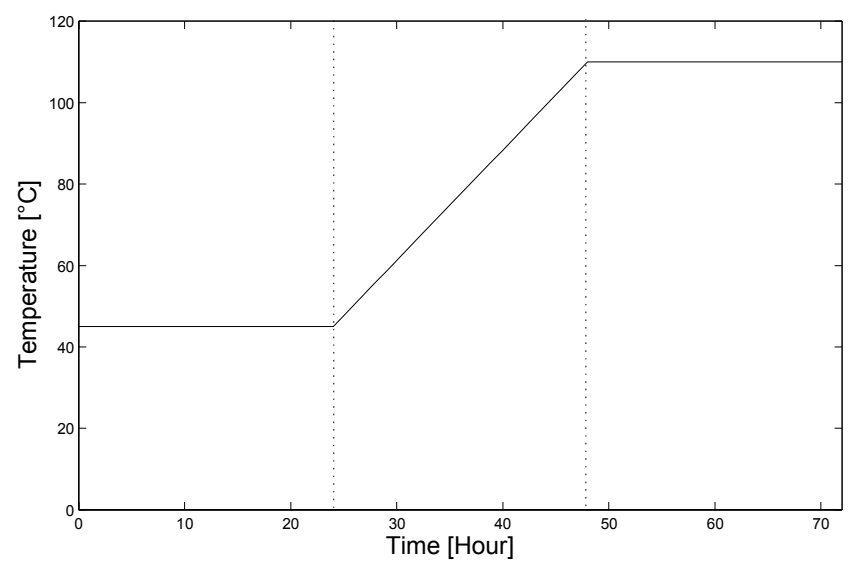

Figure 2: Drying profile of the samples

Otherwise, in order to determine the moisture of the material tested, the samples were weighted just after the compression test and after a dry cycle in an oven. The drying profile is presented on the figure 2. It can be divided in three parts. The first part, consists in a drying with a moderate temperature for 24 hours. The majority of the water contained on the external surface of the sample is evaporated. The second part of the cycle consists in an increase of the temperature to $110^{\circ} \mathrm{C}$. It is then necessary to slowly increase the temperature to avoid sample failure. Indeed, at this level, the quantity of water available in the material is still high. If the temperature rise is too fast, the pressure of the water in the centre of the sample increases and causes the break of the sample. This cannot be accepted because it will create a loss of material and accordingly, falsify the measurement of the dry weight. The last part of the curve is to reach a plateau at $110^{\circ} \mathrm{C}$ to evaporate water staying in the sample. The moisture is calculated thanks to equation 1:

$$
\mathrm{H}[\%]=\frac{\mathrm{M}_{\mathrm{m}}-\mathrm{M}_{\mathrm{ds}}}{\mathrm{M}_{\mathrm{ds}}}
$$

With $\mathrm{H}[\%]$ the moisture in percentage of dry mass, $\mathrm{M}_{\mathrm{ms}}$ the mass $(\mathrm{g})$ of the moist sample and $\mathrm{M}_{\mathrm{ds}}$ the mass $(\mathrm{g})$ of the dry sample.

\section{Constitutive behaviour}

A previous study realized on clay paste [7] allows to assume an isotropic elasto-viscoplastic behaviour. In order to model the material in its elastic range, we consider the Hooke's law :

$$
\overline{\bar{\varepsilon}}_{\mathrm{E}}=\frac{1+v}{\mathrm{E}} \overline{\bar{\sigma}}-\frac{v}{\mathrm{E}} \operatorname{tr}(\overline{\bar{\sigma}}) \overline{\overline{\mathrm{I}}}
$$

With E the Young's Modulus in MPa, $v$ the Poisson's ration, $\overline{\bar{\varepsilon}}_{\mathrm{E}}$ the elastic strain tensor and $\overline{\bar{\sigma}}$ the stress tensor.

In the visco-plastic range, we use a Norton-Hoff constitutive equation.

$$
\overline{\overline{\mathrm{S}}}=2 \mathrm{~K}\left(\sqrt{3} \dot{\bar{\varepsilon}}_{\mathrm{VP}}\right)^{\mathrm{m}-1} \dot{\overline{\bar{\varepsilon}}}_{\mathrm{VP}}
$$


With $K$ the material consistency in $\mathrm{MPa}, \mathrm{m}$ the strain rate sensitivity parameter, $S$ the deviatoric stress tensor, $\dot{\bar{\varepsilon}}_{V P}$ the strain rate tensor, $\dot{\bar{\varepsilon}}_{V P}$ the équivalent strain defined by:

$$
\dot{\bar{\varepsilon}}_{\mathrm{VP}}=\sqrt{\frac{2}{3} \overline{\bar{\varepsilon}}_{\mathrm{VP}}: \overline{\bar{\varepsilon}}_{\mathrm{VP}}^{\cdot}}
$$

In this study, we suppose that the limit between the elastic range to the viscoplastic range can be modelled by the Von Mises plasticity criterion.

\section{Results and discussion}

\section{Different parameters identification}

The different parameters of the rheological laws were identified by inverse analysis based on the experimental force / displacement curves. The commercial software Forge 2009 was used to perform the parameters identification.
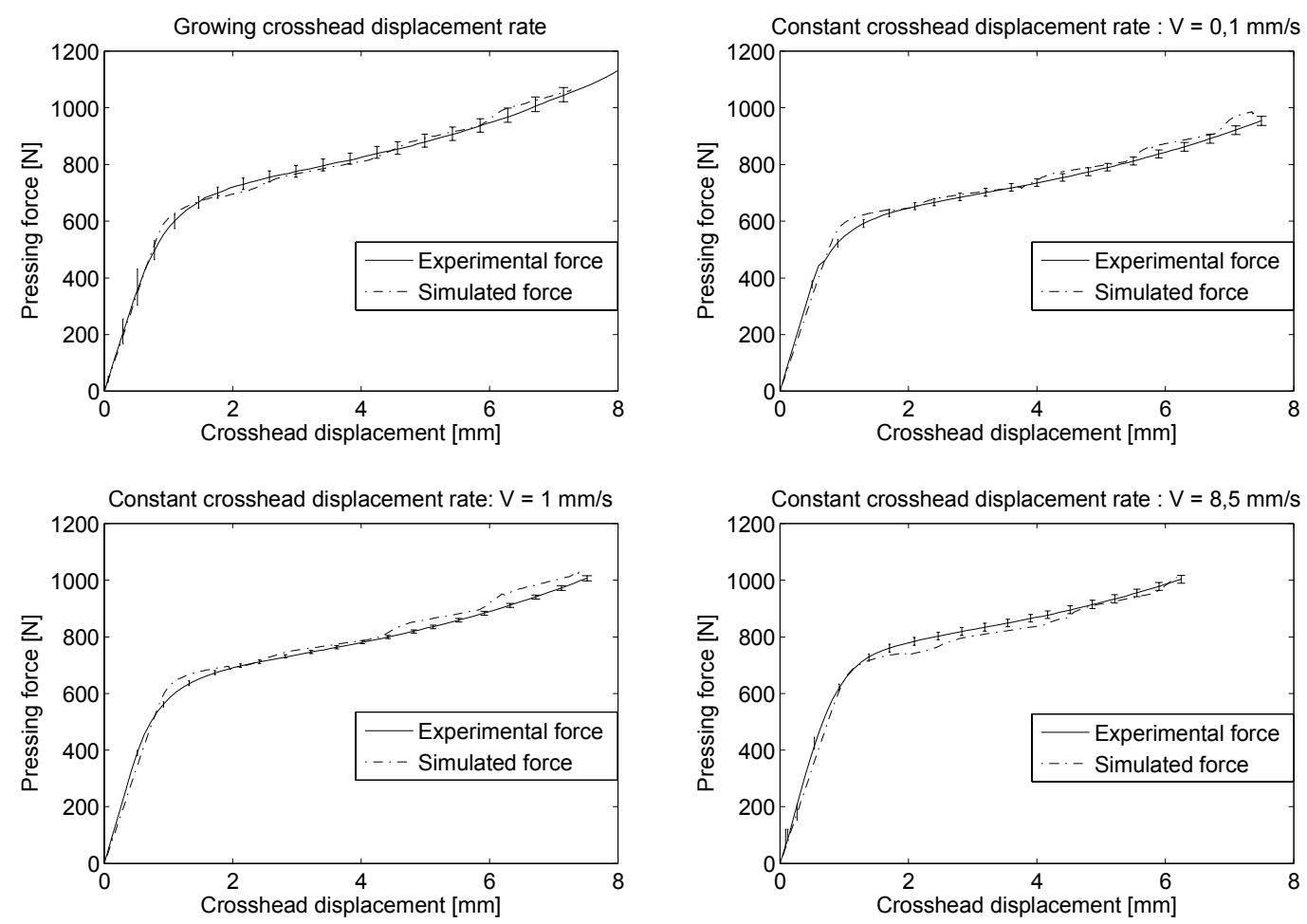

Figure 3: Comparison between the experimental/numerical compression load.

The figure 3 shows a comparison between the experimental and the numerical force / displacement curves for the four paths of compression. The curves of the graphs on the top left corner represent the variation of the pressing force according to the displacement for the compression test with an increasing crosshead displacement rate. The different parameters of the behaviour's laws were determined by an inverse analysis using the average experimental curve of five tests in such conditions. The error bar represents the minimal and the maximal values measured during the tests. We note that the repeatability of the test is good. The dotted curve shows the pressing force obtained by numerical simulation using the fitted parameters. The used of an automatic remeshing during the simulation, create some non-linearity on the compression load. The three other curves represent the pressing force of the compression tests performed with a constant crosshead 
displacement. The comparison of the curves highlights a good agreement between the simulation and the experimental. The numerical model captures the variation of the pressing force with a good accuracy (less than six percent of mean square error).

\section{Effect of the moisture}

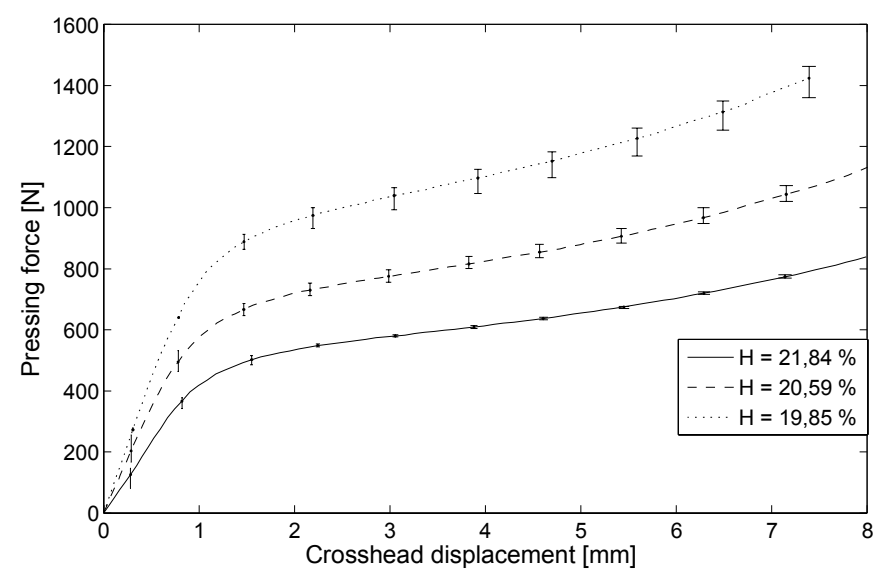

Figure 4: Compression load for three values moisture

The figure 4 shows the variation of the compression load according to the displacement of the upper tools for different samples' moisture. At first sight, the rheological behaviour of the clay paste depends on the moisture. We can see that an increase of two percent (in percent of dry weight) allows to decrease by half the force during the compression test.
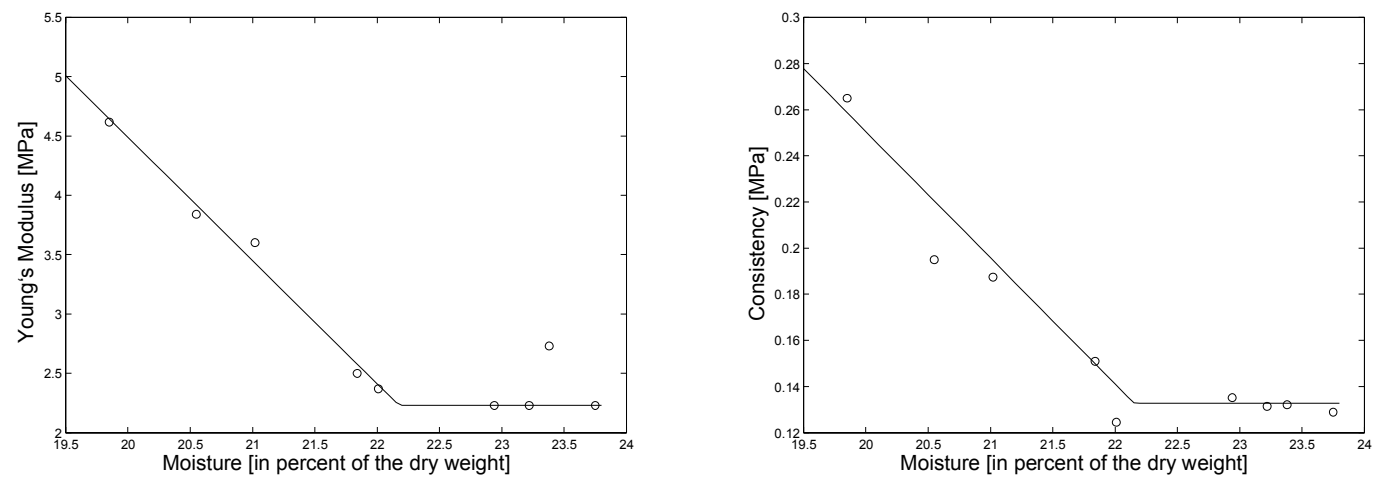

Figure 5: Evolution of the constitutive parameters versus the moisture.

The figure 5 shows the evolution of the Young's modulus and the consistency according to the moisture of the clay paste. The variation for both parameters is almost linear up a critical value of moisture $\mathrm{H}_{\text {lim }}=22,15 \%$ of the dry weight. When the moisture becomes higher than this limit value, a saturation phenomenon occurs. Indeed, the Young's modulus and the consistency do not change anymore. The moisture has a minor influence on the parameter of sensitivity to the strain rate. This effect can thus be neglected.

\section{Numerical application}

For confidentially reasons, we cannot perform a real industrial application in this paper. However, we propose an application on a simplified geometry with stress concentration occurring during the pressing of tiles. We modelled a compression test involving the shaping of a tile lug, an area frequently subjected to defects. The figure 6 shows the tools used to form the tile lug. 


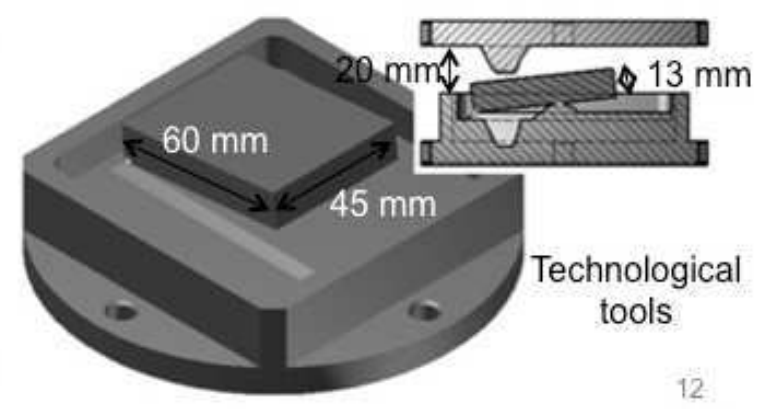

Figure 6: Tools and sample for the simplified compression test

The numerical simulations of this compression test, performed using Forge 2009, will be helpful to understand the influence of the moisture on the defect appearance during the pressing. To simulate the cracks, we used an elements deletion algorithm based on a limit value of the Latham and Cockroft Criterion [10]:

$$
C_{\mathrm{LC}}=\int_{0}^{\bar{\varepsilon}_{\mathrm{R}}} \frac{\operatorname{Max}\left(\sigma_{\mathrm{I}}, \sigma_{\mathrm{II}}, \sigma_{\mathrm{III}}\right)}{\bar{\sigma}} \mathrm{d} \bar{\varepsilon}
$$

When the value of the criterion of an finite element becomes higher than 0,3 this element is deleted allowing to simulate a crack occurrence and its growth. [7]

The figure 7 provides a comparison of the computed Latham and Cockroft criterion and the first principal stress field for two materials A and B. The material A contains 23 percent of the dry weight of water and the material B contains 20,5 percent of water. We can see on the left hand side figures that the moisture had a poor influence on the damage criterion. The volume deleted during the simulation is similar for both materials. On the other hand, the right hand side figures show that the moisture strongly influences the first principal stress field. The range of the first principal stress doubles when the moisture increases by 2.5 percent. The moisture affects the formability of the tile. Indeed, the force, required to press the tile, increases rapidly, when the moisture of the material decreases.

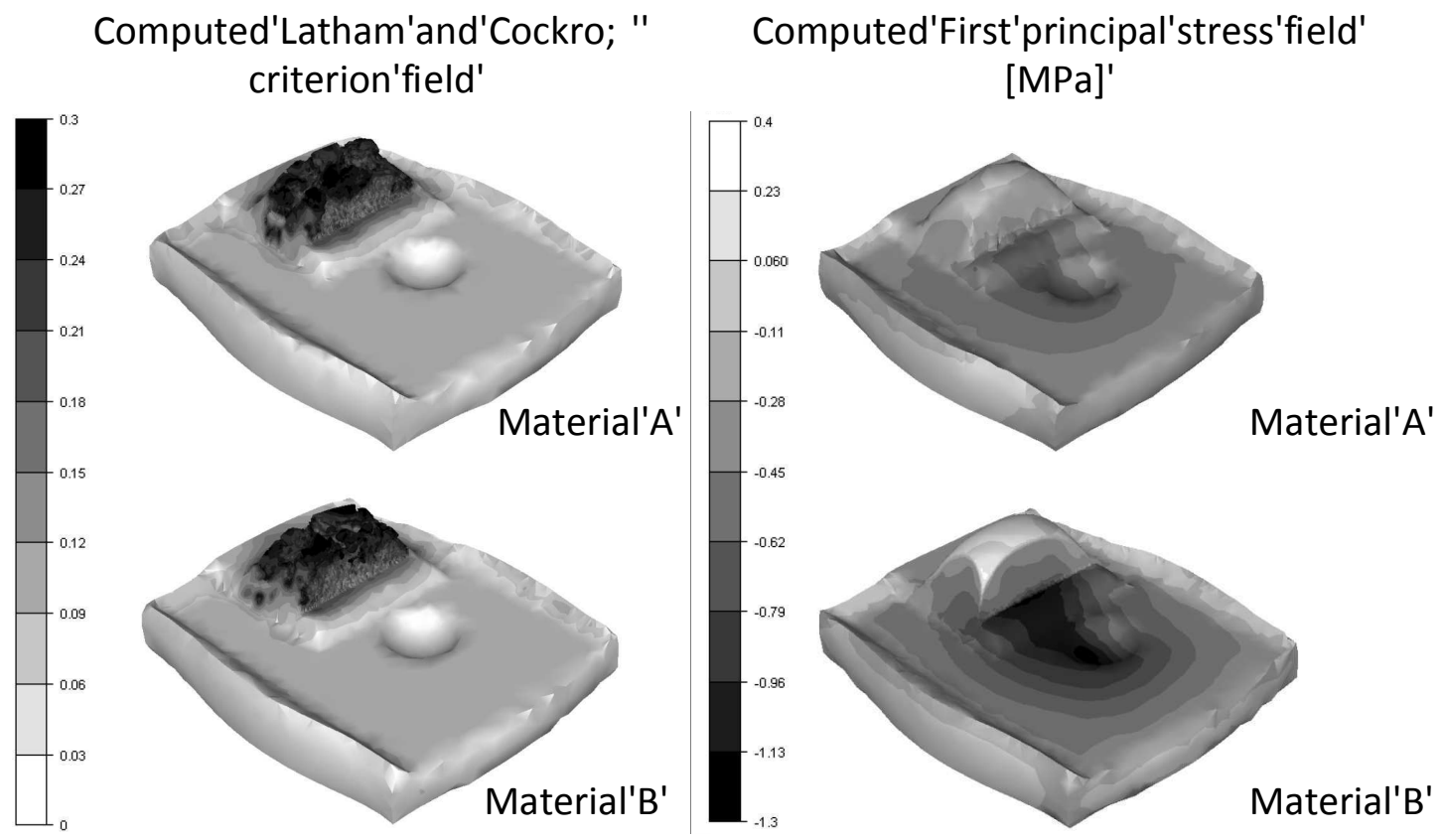

Figure 7: Von Mises stress field for two materials 


\section{Conclusion}

This study has highlighted the influence of the moisture on the rheological behaviour of a clay paste. The tests used to identify the rheological parameters were some based on free compression tests perform with four crosshead displacement paths. We identified the parameter of an elastovisco-plastic behaviour for the extruded clay paste. The moisture influences essentially the Young's modulus and the consistency of the materials. We can models it using a linear interpolation. We could observe that a small variation of the moisture created a strong variation of the rheological parameters. A increase of 2.5 percent of the moisture allows to halve the Von Mises equivalent stress. According to the numerical model, the variation of the moisture does not influence the appearance of defects (using the Latham and Cockroft criterion) on the tile lug.

\section{Acknowledgements}

The present study was supported by Terreal company. The authors wish to acknowledge this industrial partner for this support and his help.

\section{References}

[1] M. J. Ribiero, J. M. Ferreira, J. A. Labrincha, Plastic behaviour of different ceramic pastes processed by extrusion, Applied Clay Science, 31, p515-519, 2005

[2] B. Baran, T. Ertürk, Y. Sarikaya, T. Alemdaroglu, Workability test method for metals applied to examine a workability measure (plastic limit) for clays, Applied clay science, 20, P53-63

[3] C. De Oliveira Modesto, A. M. Bermandin, Determination of the clay plasticity: Indentation method versus Pfefferkorm method, Applied Clay Science, 40, p15-18, 2008

[4] F. A. de Andrade, H. A. Al-Qureshi, D. Hotza, Measuring and Modelling the Plasticity of Clays, Materials Research, 13, p395-399, 2010

[5] Y. Gayou, E. Padayodi, K. E. Atcholi, D. Mezzane, M. A. Fremy, P. Saint Gregoire, Etude comparative du comportement mécanique des matrices de quatre types d'argile, 2008

[6] E. Foudrinier, Etude numérique et expérimentale du procédé d'extrusion de pâtes argileuses, Thesis, Ecole Nationale des Mines de Paris, 2007

[7] J. Vignes, F. Schmidt, G. Dusserre, O. De Almeida, J. F. Dalmasso, Numerical simulation of clay tiles compression, Key Engineering Materials, 504-506, p1403-1408, 2012

[8] C. Djelal, Designing and perfecting a tribometer for study of friction of a concentrated clay water mixture against a metallic surface, Materials and structures, 34, p51-58, 2001

[9] F. Moore, Rheology of ceramics systems. Maclaren and Sons, London, p51-57

[10] M.G. Cockroft, D.J. Latham, Ductility and workability of metals, Journal of the institute of metals, p33-39, 1968 